\title{
El garrotillo (Difteria) en España (Siglos XVI y XVII)
}

\author{
Enrique Laval $R$.
}

The strangling angel of children (diphtheria) in Spain (XVI and XVII centuries)

En el siglo IV A.C., Hipócrates habría descrito los síntomas de asfixia y la evolución clínica de lo que pudo ser una difteria. Pensaba que con sangrías, era posible aliviar la ectasia sanguínea, que creía tener lugar en la carótida, haciendo inhalar con una paja vapor de agua, en la que añadía aceites volátiles. Pero más radical y atrevido pareció ser Praxágoras, en la segunda mitad de aquel siglo, "quien cortaba la campanilla para facilitar la respiración en caso de edema".

Es importante señalar que en el Talmud, en el siglo II D.C., se menciona la diferencia con el nombre de "Askara". Cuando se producía una epidemia, se enunciaba el peligro "con el toque de las trompetas, a continuación de la primera defunción y no después de la tercera como era lo habitual". Agrega este libro que la muerte ocurre por asfixia y "que es la peor de las 903 formas de muerte conocidas". Además manifiesta que quien come lentejas una vez al mes, echa sal a las comidas y agua a todas las bebidas mantiene la Askara alejada de su casa". Se piensa que todo esto sólo podía referirse a la difteria, especialmente al "crup".

La enfermedad, como proceso maligno de la garganta, habría sido conocida por Areteo de Capadocia, con el nombre de "úlcera siríaca o egipcia", quien describió epidemias de úlceras faríngeas, en siglo II D.C., en Siria y Egipto. En el siglo VI D.C., Aecio de Amida, médico de la corte bizantina, informa en su célebre Tetrabilion acerca de la crisis asfíctica, la parálisis del velo del paladar con la salida de líquido por la nariz al bebé. También Galeno, año 131 D.C., cita una enfermedad con expectoración y membranas. En la Edad Media, Baronius describe las epidemias que aparecieron en Roma, en los años 856 y 1004, de un catarro descendente de la

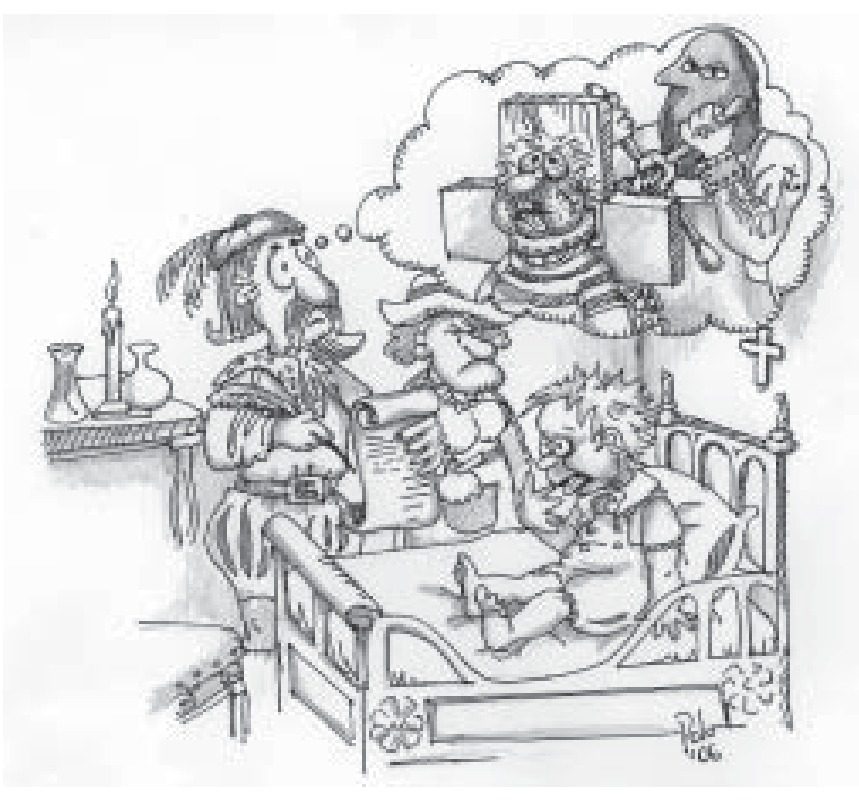

faringe, que conducía a la sofocación y que denomina "pestilencia faucium". En el siglo XIV se describe una epidemia en Inglaterra (Short, 1389); en el siglo XVI se observaron epidemias en el Rhin y en la baja Alemania. A partir de aquel siglo y principio del XVII, se extiende por España, pasando luego a Italia ${ }^{1-5}$.
Según Schreiber y Mathys, las primeras descripciones de la difteria y de la sífilis en Europa, proceden del famoso médico y escritor español Francisco López de Villalobos (1473-1549). Respecto a esta última enfermedad estarían de acuerdo Pedro Laín Entralgo, Anastasio Chinchilla y Eduardo García Real, eminentes historiadores de la Medicina Española, pero no mencionan en parte alguna "la paternidad de la descripción de la difteria", atribuida a López de Villalobos ${ }^{1,6-9}$.

Si nos atenemos a la Cronología de la Medicina y de la Higiene, en lo que corresponde a la difteria, elaborada por Garrison, la primera descripción valedera de la enfermedad habría sido efectuada por Hartman Schedel, a raíz de una epidemia acontecida en Nüremberg, Alemania, en $1492^{5}$.

La casi totalidad de los historiadores califican el siglo XVI, de "siglo español", por haberse erigido durante él, la hegemonía del mundo por España, con los monarcas de la Casa de Austria, Carlos I y Felipe II. Sin embargo, la que se pudiera llamar "Edad de Oro", de la cultura, del arte y de la ciencia española, no coincide exactamente con el siglo XVI: comprende en realidad todavía la mitad del siglo XVII.

Por lo tanto, se consideraría el lapso comprendido entre 1500 y 1665 , como la "Edad de Oro de la Medicina Española”, debida a la naturaleza, expansión y florecimiento de las fuerzas intelectuales, que desde mucho tiempo 
antes se habían venido acumulado en España y al empuje de la traducción que, en Medicina sobre todo, se remontaba a la época anterior a los Reyes Católicos.

Ofrece gran interés el señalar las grandes epidemias que han devastado España durante el siglo XVI. En efecto, han dado ocasión a grandes esfuerzos de observación clínica y a la aplicación de curiosos métodos terapéuticos por parte de los médicos españoles. Vale la pena mencionar la peste bubónica, el tifus exantemático, la viruela y muy especialmente la aparición en 1583, del "garrotillo", con cuyo nombre designaron los médicos españoles toda angina sofocante o esquinancia, en la mayoría de los casos de naturaleza diftérica, causando numerosas víctimas ${ }^{8}$.

Luis Mercado (1525?-1611), la figura más importante de la medicina renacentista española, natural de Valladolid, en sus "Consultaciones morborum complicatorum et gravisimorum", publicadas en 1613 después de su muerte, hace la primera y magistral descripción del "garrotillo", tras su aparición epidémica en España en las últimas decenas de la centuria. Pero el estudio más completo y pormenorizado es el de Juan de Villarreal ("De signis, causis, essentia, prognostico et curatione morbi soffocantis"), publicado en Alcalá de Henares en $1611^{7}$.

Así estos dos sobresalientes médicos españoles, Mercado y Villarrea -este último con gran acopio de argumentación eruditasostienen la novedad radical del "garrotillo" y lo que no parece ofrecer duda es su prioridad respecto al gran epidemiólogo francés Baillou (1538-1616), que también describió la angina cruposa a comienzos del siglo $\mathrm{XVII}^{6}$.

Los españoles daban el nombre de "garrotillo" a la angina diftérica y al crup, si bien a veces incluían las amigdalitis agudas y otras anginas malignas, a causas del aspecto vultuoso, que tomaba la cara de los enfermos y que lo hacía comparable a la de los sometidos a la pena del "garrote", porque en los que sufrían este castigo, el cordel obraba en toda la circunferencia de la garganta y lo mismo ocurría en la enfermedad en cuestión, "cuya causa dependia de una sustancia crasa y compacta a medida de membrana, que afectaba el tragadero, la garganta y las fauces, como si fuera un lazo, de tal suerte que las partes necesariamente lle- gan a juntarse, del mismo modo que se le tuerce al cuello con una soga"s.

Entre 1583 y 1638, la difteria fue "seis veces epidémica” en España, extendiéndose a Italia, en 1618. En el curso de aquellos años fue descrita por varios médicos: Casales, Fontech, Villarreal, en 1611. Posteriormente tomaron nota de ella Núñez Herrera (1615) Juan Soto (1616), Lima, Francisco de Figueroa y Lorenzo de San Millán (1616). En brotes epidémicos después de 1638, destacan las comunicaciones de Pedro Miguel de Heredia en 1665; los relatos más completos, además de este último autor, fueron seguidos por el estudio histórico de Fernández Navarrete (1764-1771) y por el tratado de Juan Antonio Pascual en 17845. Son también notables los escritos de los italianos Gian Andrea Sgambetti, Francesco Nola y Gian Batista Carnevale, en $1620^{7}$.

Todo esto muestra la importancia que tuvieron en España las sucesivas epidemias de "garrotillo", así como el impacto que provocó su aparición en Italia, donde la llamaron "pedancone maligna", "morbo sofocante", "enfermedad estrepitosa", "enfermedad anginosa estranguladora" 2 .

La "angina membranosa" se extendió por todo el mundo ocasionando pandemias mortíferas, que siguieron una periodicidad bastante regular cada 15 años: Bordeu la observó en Francia (1746), Home, en Edimburgo (1765) y Samuel Bard, en Nueva Cork (1771), conformando la identidad causal del crup con formas anginosas de la enfermedad ${ }^{10}$.

Como lo ha destacado Ulrich Friedemann, las epidemias de difteria presentan algunos rasgos característicos que siempre se repiten. Las de sarampión aumentan rápidamente y desaparecen con la misma celeridad; en cambio la difteria se extiende muy lentamente. Nunca invade bruscamente, en pocas semanas, naciones enteras o continentes, como la naturaleza. Las epidemias tienen primero carácter local, pero aparecen simultáneamente o con breves intervalos en regiones muy alejadas ${ }^{11}$.

Dijimos anteriormente que si bien Luis Mercado efectuó la primera descripción del "garrotillo", sin lugar a dudas el estudio más completo y minucioso correspondía al de Juan de Villarreal, natural de Ubeda y Doctor de la Universidad de Alcalá. Anastasio Chinchilla, citado por García Real, insiste y con razón, que Villarreal había descrito siglo y medio antes que Chisi las membranas de la angina diftérica, deduciendo importantes datos del examen de las misma; que él recomienda "como de buenos efectos, la sangría de la vena cefálica, antes que otros hayan hecho igual recomendación; en que ha propuesto los toques locales con ácido nítrico diluido, antes que Delpech y que ha compuesto su libro 154 años antes de la publicación del de Home (Edimburgo, 1765), considerado erróneamente por Desruelles, como el primer trabajo didáctico consagrado a esta enfermedad; siendo igualmente falsa la opinión de aquel de que la angina membranosa hubiera sido desconocida en España, que padeció epidemias muy bien observadas por sus médicos en los años 1583, 1596, 1600 y 1605".

Juan de Villarreal divide su obra en dos libros: el primero está subdividido en nueve capítulos, tratando primero de la etimología de la enfermedad, declarándose contrario a la denominación de "morbos suffocativus sive strangulatorious", puesto que otras enfermedades, como la "pleuritis, pulmonía, aplopegía, asma, etc solían quitar la vida, impidiendo la respiración y por consiguiente sofocando, y sin embargo, a nadie se le había ocurrido llamarles enfermedades sofocantes". Prueba que tampoco podía aplicarse la denominación de "morbos strangulatorius", porque para esto, era necesario que la estrangulación fuera acompañada de la luxación de las vértebras del cuello, lo cual no sucedía así, por lo que prefiere el nombre de "garrotillo", de dar "garrote".

En el segundo capítulo discute si esta enfermedad fue "conocida de los antiguos $y$ aún modernos, negando en forma tajante que Hipócrates, Areteo, Celio, Aureliano y Aecio hubieran escrito algo semejante". Vuelve a insistir que la causa de esta enfermedad "no eran aftas, ni úlceras ni carbunclos sino una membrana como un pergamino, de tal consistencia, que si extendia con las manos, se alargaba y se encogía como si estuviera humedecido, cuya experiencia había aprendido en los vivos por pedazos de membrana que arrojaban y en los muertos por medio de la disección anatómica, añadiendo que había visto miles de enfermos"9.

Luego dice que esta enfermedad es con- 
tagiosa "fomite et per contactum, non vero ad distans". Prueba que es contagiosa porque vio familias enteras padecer este mal, empezando por uno e invadiendo a los demás. Por contacto porque "vio niños que padeciendo de este mal lo pegaron a sus madres lactando y, por el contrario, madres enfermas que transmitieron a sus hijos; por fomes o infección por haber visto presentarse la enfermedad en personas que durmieron en la cama de los enfermos; pero no "ad distans", porque dependiendo esta enfermedad de una materia crasísima, membranosa, seca y consistente no era apta para propagarse por hálito, diseminarse por el aire y marchar a larga distancia".

Los signos de esta enfermedad añade Villarreal, "no siempre se presentan del mismo modo: abierta la boca y deprimida la lengua, unas veces se notaba un ápice enteramente blanco que salia de lo hondo de la garganta y que impedia la deglución; otras, cierta costra, como una membrana que ceñia las fauces, no perfectamente blanca, sino declinado a lívida, cuya variedad nace de la diferencia de las cusas. Aunque la lengua puede presentase blanca y no sea un síntoma propio y patognomónico de esta enfermedad, sin embargo, existiendo al mismo tiempo la dificultad de tragar y la epidemia reinante, puedes estar bien seguro de que esta enfermedad es el garrotillo. Tendrás más certeza todavía, si vieses tumores en el cuello o detrás de las orejas, cuyos tumores se observan en todos y aun más en aquellos cuya costra declina a livida y es como membrana". Diríamos que esta es una casi perfecta descripción de la angina diftérica, escrita en el siglo XVII y que nos recuerda lo que hemos observado en años recientes. Demostró Villarreal "que a este proceso no siempre acompañaban inflamación, dolor ni calentura. Esto podía suceder porque la malignidad del mal extinguía desde el principio el calor vital'.

Respecto de la región afecta principalmente, opina que es en primer lugar, la "garganta" (laringe) y a continuación el "tragadero" (faringe).

Villarreal afirma que esta enfermedad ataca con mayor frecuencia a los niños, después a los jóvenes, más rara vez a los adul- tos y jamás a los viejos. El sexo femenino se ve perfectamente comprometido. Da mucha importancia en el pronóstico al color de la membrana, asegurando que si es blanca es mucho menos grave que cuando tiene un tinte lívido y es gravísimo si su color es negro. Si la membrana aparece ya formada, el pronóstico es mucho peor que si sólo se aprecia un "pedacillo o ápice como un pitoncillo blanco". En este proceso no puede haber verdaderamente crisis a la salud ni a la muerte, si por crisis se entiende la traslación súbita de la materia de una parte a otra, "porque ya formado no puede trasladarse por su crasitud y adherencia, teniendo que terminar o por la muerte por estrangulación o por la destrucción de las membranas por los medicamentos". La hemorragia o flujo sanguíneo nasal o bucal es un signo mortal, "pues no ha visto salvarse a ningún enfermo".

Aconseja administrar al principio "alimentos muy nutritivos, porque en esta enfermedad al comienzo suelen desfallecer tanto las fuerzas, que los dolientes quedan como exánimes, con sus extremidades frías". Es conveniente la administración del vino, cuando los enfermos van perdiendo sensiblemente las fuerzas.

Rechaza como inútiles y hasta perjudiciales las sangrías en las venas sublinguales, así como los eméticos, ya que sólo hacen arrojar las materias estomacales y no " $l a$ materia adherida a la garganta". Villarreal ha renunciado al empleo del mercurio en fricciones, por considerarlo peligroso. No cree conveniente la causticidad ni la cauterización de la membrana, "porque aumentando la sequedad y contracción de las partes, engruesan las membranas e irritan más las úlceras". Las tentativas de extracción no hacen más que irritar las costras y además resultan inútiles, porque no pudiendo extraerse más que un trozo, volverá a reproducirse inmediatamente.

Pueden utilizarse al comienzo los gargarismos repercusivos y fríos, pero una vez que haya aparecido la membrana, se preferirán los emolientes suaves y templados.

Anastasio Chinchilla concluye "que si bien Juan de Villarreal no conocia, naturalmente, el microscopio, ni la microbiolo- gía, ni los sueros ni las vacunas, en todo de la experiencia clínica, parece por completo un escultor moderno". Este gran médico español empezó sus observaciones en Andalucía los años 1590 y 1591, para continuarlas hasta 1608 , en que escribió su obra ${ }^{8,9}$.

Finalmente, es preciso recordar que fue Pierre Philippe Bretonneau, en 1826, quien demostró definitivamente que la angina diftérica y la forma laríngea, el crup (palabra onomatopéyica que recuerda al graznido del cuervo) tienen una causa común.

La llamo "difteritis", que significa inflamación membranosa. Trousseau describió el cuadro toxico de la afección, probando su producción independiente del grado de inflamación membranosa, cambiando aquel nombre, por el de difteria ${ }^{2}$.

\section{Referencias}

1.- Schreiber W, Mathys F K. Infectio. Historia de las enfermedades Infecciosas. Ed. Roche. Basilea. Suiza.1987. p 175-9.

2.- Pons A P. Patología y Clínica Médicas. Tomo VI. Enfermedades Infecciosas.. Ed. Salvat. Barcelona. España. 1952. pp 316-31

3.- Brugsch T H. Tratado de Patología Médica. Tomo I. Ed. Labor. Barcelona. España. 1937. p 415-30.

4.- Castiglioni A. Historia de la Medicina. P. 537. Ed. Salvat. Barcelona. España. 1941

5.- Garrison F H. Historia de la Medicina.. Ed. Interamericana. México. 1966. p 175, 62144

6.- Laín Entralgo P. Historia de la Medicina Moderna y Contemporánea. P. 39 Ed. Científico Médica. Barcelona. España. 1954.

7.- Laín Entralgo P. Historia Universal de la Medicina. Ed. Salvat. Madrid. España. 1975. Tomo III p 32, Tomo IV p 37.

8.- García Real E. Historia de la Medicina en España. Ed. Reus. Madrid. España. 1921. p 53, 244-54.

9.- Chinchilla A. Historia de la Medicina Española. Tomo II. Impta. De D. José Mateu Cervera. Valencia. España. 1845. p 287-301.

10.- Bariéty M, Coury C H. Histoire de la Médecine. Ed. A. Fayard. Paris. Francia. 1963. p 560-1.

11.- Bergmann G V, Staehelin R. Tratado de Medicina Interna. Enfermedades Infecciosas. Tomo I. Ed. Labor. Barcelona. España. 1942. p 525-76. 